\title{
Georges de Rham et le problème d'homéomorphie des rotations
}

\author{
Manuel Ojanguren et Jérôme Scherer*
}

\section{Introduction}

Georges de Rham devint immédiatement célèbre avec sa thèse (rédigée entre 1928 et 1930, publiée en 1931, [5]), dans laquelle il démontrait deux conjectures d'Élie Cartan. Mais, avant même de commencer à travailler à sa thèse, il s'intéressa aux variétés de dimension 3 , en particulier aux espaces lenticulaires (dont il sera question plus loin) et à un problème lié à leur classification, celui de l'homéomorphie des rotations de la sphère, qu'on peut formuler ainsi:

Soient $R_{1}$ et $R_{2}$ deux rotations de la sphère $S^{n}$. Supposons qu'il existe un homéomorphisme $f$ de $S^{n}$ tel que $R_{2}=f R_{1} f^{-1}$. Est-ce qu'il existe alors une isométrie $T$ de $S^{n}$ telle que $R_{2}=T R_{1} T^{-1}$ ?

De Rham parle de ses travaux, ainsi que de ceux de Reidemeister et Franz, au congrès de topologie de Moscou en 1935, [6]. Des progrès sont faits dans les années qui suivent, mais ils n'apportent que des réponses partielles et ce n'est qu'au milieu des années 60 que de Rham publie [7], où il résout positivement la question dans le cas où $f$ est un difféomorphisme. Il revient encore une fois sur ce sujet, alors qu'il a déjà pris sa retraite depuis une dizaine d'années, pour en faire une synthèse dans un bel article historique, [9], motivé par la réponse surprenante donnée par Cappell et Shaneson, [3], 45 ans après la conférence de Moscou. Nous recommandons au lecteur d'y jeter un coup d'œil et décidons, quant à nous, de faire partager, en petite dimension, l'essence de cette question qui poursuivit de Rham tout au long de sa vie.

\section{Homéomorphie dans $\mathbb{R}^{n}$}

Pour mieux comprendre la nature du problème, nous pouvons commencer par une question légèrement différente. Soient $A$ et $B$ deux matrices réelles $n \times n$ et supposons qu'il existe un difféomorphisme ou un homéomorphisme $f: \mathbb{R}^{n} \rightarrow \mathbb{R}^{n}$ tel que $B=f A f^{-1}$. Peut-on

\footnotetext{
* Le deuxième auteur bénéficie du soutien du projet FEDER/MEC MTM2007-61545.
} 
en conclure que $A$ et $B$ sont semblables, c'est-à-dire qu'il existe une matrice $S$ telle que $B=S A S^{-1}$ ?

Il n'y a pas de différence entre le cadre linéaire et différentiable (via la dérivée du difféomorphisme $f$ ), mais la richesse de ce problème dans le cadre topologique est déjà illustrée dans le cas des applications linéaires de $\mathbb{R}$ dans $\mathbb{R}$ (tiré de l'excellent article [11] de Kuiper et Robbin). Concrètement, la multiplication par 2 et par 8 sont des applications linéaires homéomorphes via $\phi(x)=x^{3}$. En effet si $f(x)=2 x$ et $g(x)=8 x$, alors

$$
\phi \circ f \circ \phi^{-1}(x)=(2 \sqrt[3]{x})^{3}=8 x=g(x) .
$$

De fait il y a exactement sept classes d'homéomorphie d'applications linéaires de $\mathbb{R}$ dans $\mathbb{R}$ ! Trois classes ne contiennent qu'un seul endomorphisme, il s'agit de l'application linéaire nulle, de l'identité et son opposé. Les quatre autres classes sont données par les composantes de $\mathbb{R} \backslash\{-1,0,1\}$, c'est-à-dire les intervalles ]- $-, 1[]-1,,0[$, ]0,1[ et ] $1, \infty[$. En effet, la conjugaison par un homéomorphisme ne change pas le signe d'une valeur propre, et si $f(x)=a x$ avec $0<|a|<1$, alors $f^{n}(x)$ tend vers zéro lorsque $n$ tend vers l'infini et cette propriété aussi est préservée par conjugaison.

Théorème 1.1 (Kuiper et Robbin, [11]) Les applications linéaires $f(x)=$ ax et $g(x)=$ $b x$ de $\mathbb{R}$ dans $\mathbb{R}$ sont homéomorphes si et seulement si a et b appartiennent tous deux à l'un des sept sous-ensembles de $\mathbb{R}$ suivants: $\{0\},\{1\},\{-1\},]-\infty,-1[]-1,,0[] 0,,1[$ et $] 1, \infty[$.

Pour retourner au problème sur lequel travailla de Rham, nous nous restreignons aux applications linéaires de $\mathbb{R}^{n+1}$ qui fixent la sphère unité $S^{n}$.

\section{Le cas du cercle}

Considérons deux rotations du cercle (le cercle unité dans $\mathbb{R}^{2}$ ), l'une $\rho_{\alpha}$ d'angle $\alpha$, l'autre $\rho_{\beta}$ d'angle $\beta$. Si ces rotations sont isométriques, les matrices des deux rotations sont semblables. Elles doivent en particulier avoir la même trace, si bien que $\alpha= \pm \beta$ (une symétrie axiale conjugue $\rho_{\alpha}$ en $\rho_{-\alpha}$ ). Nous aimerions maintenant savoir quand deux telles rotations sont homéomorphes, c'est-à-dire quand il existe un homéomorphisme $f: S^{1} \rightarrow S^{1}$ qui conjugue l'une en l'autre:

$$
f^{-1} \circ \rho_{\alpha} \circ f=\rho_{\beta} .
$$

La solution de ce problème est la conséquence d'un résultat plus général de Poincaré. Effectuons une simplification qui nous permettra de supposer que l'homéomorphisme qui conjugue une rotation en l'autre fixe un point, disons $(1,0)$.

Lemme 2.1 Supposons que $\rho_{\alpha}, \rho_{\beta}: S^{1} \rightarrow S^{1}$ sont conjugués par $f$ et que $f(1,0)=$ $(\cos \gamma, \sin \gamma)$. Définissons $g=\rho_{-\gamma} \circ f$. Alors $g$ aussi conjugue $\rho_{\alpha}$ en $\rho_{\beta}$ et $g(1,0)=$ $(1,0)$

Démonstration. On a $\rho_{\alpha} \circ g=\rho_{\alpha} \circ \rho_{-\gamma} \circ f=\rho_{-\gamma} \circ\left(\rho_{\alpha} \circ f\right)=\rho_{-\gamma} \circ\left(f \circ \rho_{\beta}\right)=g \circ \rho_{\beta}$. 
Démontrons maintenant le résultat sur les rotations du cercle. On suppose que les rotations $\rho_{\alpha}$ et $\rho_{\beta}$ sont conjuguées par un homéomorphisme $f: S^{1} \rightarrow S^{1}$. Autrement dit, $\rho_{\alpha} \circ f=f \circ \rho_{\beta}$. Cette écriture nous permet d'éviter d'utiliser l'inverse de $f$ et nous allons de fait montrer un résultat légèrement plus général. Rappelons que le degré d'une application $f: S^{1} \rightarrow S^{1}$ est le "nombre de tours du cercle" que l'on effectue en parcourant $f$, si bien qu'une application de degré 1 peut être déformée continûment en l'identité. Plus précisément, on peut relever $f$ en une fonction réelle $F: \mathbb{R} \rightarrow \mathbb{R}$ via la projection exponentielle $\pi: \mathbb{R} \rightarrow S^{1}$ définie par $\pi(t)=e^{i t}=(\cos t, \sin t)$. Lorsque $f$ est un homéomorphisme on définit $F$ en faisant correspondre à tout $t \in[0,2 \pi$ [ le seul élément $s$ dans $[0, \pm 2 \pi$ [ tel que $\pi(s)=f(\pi(t))$. Le signe vient du fait qu'on peut parcourir le cercle soit dans le sens trigonométrique (degré 1), soit dans celui des aiguilles d'une montre (degré -1$)$. On prolonge ensuite $F$ sur tout $\mathbb{R}$ via $F(t+2 k \pi)=F(t)+2 d k \pi$. Ici $d= \pm 1$, mais en général le degré de $f$ est un entier $d$ arbitraire.

Théorème 2.2 Soit $\alpha$ et $\beta$ deux angles. On suppose qu'il existe une application continue $f: S^{1} \rightarrow S^{1}$ de degré \pm 1 telle que $\rho_{\alpha} \circ f=f \circ \rho_{\beta}$. Alors $\alpha= \pm \beta$.

Démonstration. Quitte à composer avec une rotation, on peut supposer que $f$ fixe le point $(1,0)$. Puisque la rotation $\rho_{\alpha}$ se relève sur $\mathbb{R}$ en $x \mapsto x+\alpha$, notre équation de conjugaison se convertit en

$$
F(t)+\alpha+2 c \pi=F(t+\beta)
$$

pour un entier fixé $c$ (les fonctions $F(t)+\alpha$ et $F(t+\beta)$ diffèrent d'un multiple de $2 \pi$ puisqu'elles coïncident sur le cercle, et par continuité, ce multiple ne peut être que constant). Nous adaptons maintenant l'idée de Poincaré du "nombre de rotation". Choisissons pour tout entier $n$, un entier $x_{n}$ tel que $0 \leq x_{n} \beta-2 n \pi \leq 2 \pi$. Par conséquent la suite $\left(\frac{2 n \pi}{x_{n}}\right)$ converge vers $\beta$. Or par définition du relèvement $F$ on a

$$
F\left(x_{n} \beta-2 n \pi\right)=F\left(x_{n} \beta\right) \pm 2 n \pi=x_{n} \alpha+2 c x_{n} \pi \pm 2 n \pi
$$

où la dernière égalité provient de l'équation de conjugaison appliquée $x_{n}$ fois. En divisant par $x_{n}$ on obtient ainsi que $\alpha+2 c \pi \pm \frac{2 n \pi}{x_{n}}=\frac{F\left(x_{n} \beta-2 n \pi\right)}{x_{n}}$, terme qui tend vers zéro. En d'autres termes, à un multiple de $2 \pi$ près, $\alpha= \pm \beta$.

\section{Le cas de la sphère}

Une matrice de rotation de la sphère $S^{2}$ est semblable à la matrice de rotation d'axe nordsud de la forme

$$
R_{\alpha}=\left(\begin{array}{ccc}
\cos \alpha & -\sin \alpha & 0 \\
\sin \alpha & \cos \alpha & 0 \\
0 & 0 & 1
\end{array}\right)
$$

pour un angle $0 \leq \alpha<2 \pi$. Quitte à conjuguer par une rotation, on peut donc supposer que les deux rotations considérées partagent le même axe, disons nord-sud. 
Théorème 3.1 Soit $\alpha$ et $\beta$ deux angles. On suppose qu'il existe un homéomorphisme $f: S^{2} \rightarrow S^{2}$ tel que $f^{-1} \circ R_{\alpha} \circ f=R_{\beta}$. Alors $\alpha= \pm \beta$.

Démonstration. Puisque les pôles sont les deux seuls points de $S^{2}$ fixés par les deux rotations, nous voyons que $f$ aussi fixe les pôles $N$ et $S$. Soit $p: S^{2}-\{N, S\} \rightarrow S^{1}$ l'application continue qui envoie un point de la sphère (différent des pôles) sur le point de l'équateur se trouvant sur le même méridien. On constate alors que $p$ et $R_{\beta}$ commutent ( $\left.p \circ R_{\beta}=R_{\beta} \circ p\right)$ puisque cette rotation transforme méridiens en méridiens. Par conséquent, nous avons:

$$
p \circ f \circ R_{\alpha}=p \circ R_{\beta} \circ f=R_{\beta} \circ p \circ f .
$$

Autrement dit, l'application $p \circ f$ restreinte à l'équateur, qui n'est généralement pas un homéomorphisme de $S^{1}$, mais toujours une application de degré \pm 1 , conjugue la rotation du cercle d'angle $\alpha$ en une rotation d'angle $\beta$. Le Théorème 2.2 s'applique et nous concluons que $\alpha= \pm \beta$.

\section{Les sphères de dimension supérieure}

Le cas des rotations de $S^{n}$ se réduit sans trop de difficulté (voir par exemple [7]) au cas où les rotations sont $d$ 'ordre fini. Un cas particulièrement intéressant est celui où l'action des rotations est libre, ce qui n'est possible que si la dimension de la sphère est impaire. Le cas de $S^{3}$ est déjà très intéressant, et a joué un rôle important dans le développement de la topologie algébrique, indépendamment du problème de l'homéomorphisme des rotations.

Dans sa note autobiographique [8], de Rham raconte comment il s'était intéressé aux variétés (compactes) de dimension 3 et à la conjecture de Poincaré, qui est maintenant devenue le Théorème de Perelman: La sphère $S^{3}$ est topologiquement caractérisée, parmi les variétés closes à trois dimensions, par le fait que son groupe fondamental se réduit à l'identité. Plus généralement, on pouvait se demander si la connaissance du groupe fondamental suffisait pour caractériser topologiquement une variété à trois dimensions. De Rham étudia à Paris une note d'Alexander de 1919, [1], qui exhibe deux variétés de dimension trois (des espaces lenticulaires en fait) dont les groupes fondamentaux sont cycliques d'ordre cinq.

Les espaces lenticulaires, introduits par Tietze, [13], peuvent s'obtenir de la façon suivante. On représente la sphère de dimension 3 comme un sous-ensemble de $\mathbb{C}^{2}$ :

$$
S^{3}=\left\{\left.\left(z_{1}, z_{2}\right)|| z_{1}\right|^{2}+\left|z_{2}\right|^{2}=1\right\}
$$

on choisit deux entiers naturels $p$ et $q$ premiers entre eux, et on fait agir le groupe cyclique $\mu_{p}$ des racines $p$-ièmes de l'unité en décrétant que si $\zeta=e^{2 \pi i / p}$, alors

$$
\zeta\left(z_{1}, z_{2}\right)=\left(\zeta z_{1}, \zeta^{q} z_{2}\right)
$$

Le quotient $S^{3} / \mu_{p}$ est l'espace lenticulaire $L(p, q)$. L'action de $\mu_{p}$ est libre et préserve l'orientation, donc $L(p, q)$ est une variété orientable de dimension 3, dont le groupe fondamental est cyclique d'ordre $p$. Ses groupes d'homologie entière sont $H_{0}=H_{3}=\mathbb{Z}$, $H_{1}=\mathbb{Z} / p \mathbb{Z}, H_{2}=0$. Alexander avait démontré que $L(5,1)$ et $L(5,2)$ ne sont pas 
homéomorphes, tout en ayant la même homologie et le même groupe fondamental. La démonstration utilise les coefficients d'entrelacement d'un générateur de $H_{1}$.

Dans son premier article, [4], de Rham généralise aux variétés de dimension plus grande l'utilisation des coefficients d'entrelacement comme invariants topologiques permettant de les distinguer. La définition des espaces lenticulaires de dimension 3 admet une généralisation immédiate en dimension impaire $2 n-1$ quelconque. Soit $S^{2 n-1}=\left\{\left(z_{1}, \ldots, z_{n}\right) \in\right.$ $\left.\left.\mathbb{C}^{n}|| z_{1}\right|^{2}+\ldots+\left|z_{n}\right|^{2}=1\right\}$. On choisit ensuite un entier $p$ et des entiers $l_{1}, \ldots, l_{n}$ tous premiers à $p$, et on fait agir le groupe $\mu_{p}$ sur $S^{2 n+1}$ en définissant

$$
\zeta\left(z_{1}, \ldots, z_{n}\right)=\left(\zeta^{l_{1}} z_{1}, \ldots, \zeta^{l_{n}} z_{n}\right) .
$$

L'espace quotient $S^{2 n-1} / \mu_{p}$ est un espace lenticulaire généralisé, que de Rham note $\bar{C}\left(l_{1}, \ldots, l_{n}\right)$. Étant donnés $\bar{C}\left(l_{1}, \ldots, l_{n}\right)$ et $\bar{C}\left(l_{1}^{\prime}, \ldots, l_{n}^{\prime}\right)$, leur homéomorphie équivaut à celle des rotations qui ont comme valeur propres $\left(\zeta^{l_{1}}, \bar{\zeta}^{l_{1}}, \ldots, \zeta^{l_{n}}, \bar{\zeta}^{l_{n}}\right)$, respectivement $\left(\zeta^{l_{1}^{\prime}}, \bar{\zeta}^{l_{1}^{\prime}}, \ldots, \zeta^{l_{n}^{\prime}}, \bar{\zeta}^{l_{n}^{\prime}}\right)$

Dans le dernier chapitre de sa thèse [5], de Rham donne, sans démonstration, un critère suffisant pour que deux espaces lenticulaires de dimension $2 n-1$ soient homéomorphes: À l'ordre et au signe près, les nombres $l_{i}$ et $l_{i}^{\prime}$ doivent être égaux modulo $p$ (voir [6] pour cette formulation). Le 10 avril 1935, Reidemeister lui écrit pour avoir des précisions sur ce critère et lui annoncer la résolution complète du cas de dimension 3 . Entre autre il écrit ceci:

Ich wäre Ihnen sehr dankbar, wenn Sie mir andeuten könnten, wie man die Aequivalenz dieser Räume $\bar{C}(l)$ und $\bar{C}\left(l^{\prime}\right)$ einsehen kann. Denn ich hoffe andererseits zeigen zu können, daß die symmetrischen Funktionen $f_{i}(l)$ eines Raumes $\bar{C}(l)$ nur den Abänderungen unterliegen die zugelassen werden. Für Linsenräume - also solche der Dimension drei - ist der Beweis bereits durchgeführt.

De Rham commence par lui donner les éclaircissements requis (une dizaine de pages!), puis, Reidemeister lui envoie la résolution du cas de dimension 3, pour laquelle il introduit un nouvel invariant qui portera son nom. Dans une deuxième lettre, datée du 18 mai 1935, de Rham lui dit:

Permettez moi de vous féliciter très vivement de l'envoi de vos travaux. Votre travail sur les "Linsenräume", en particulier, m'a intéressé au plus haut point et a suscité en moi une grande admiration. En l'étudiant, j'ai réussi à mettre votre méthode sous une forme qui s'applique au problème relatif aux complexes $\bar{C}\left(l_{1}, \ldots, l_{v}\right)$ au sujet duquel vous m'écriviez et que vous espériez résoudre bientôt - peut-être l'avez-vous aussi résolu maintenant? -

En effet, Reidemeister avait résolu le problème en collaboration avec Franz, d'abord dans le cas d'un groupe fondamental d'ordre une puissance d'un premier, peu après dans le cas général, [12]. La démonstration nécessite un résultat de théorie des nombres - démontré par Franz, [10] - que de Rham, par erreur, avait cru déjà connu. 
De Rham va continuer à étudier le problème des rotations d'une sphère, et surtout à essayer de démontrer que deux rotations quelconques (qu'on peut supposer d'ordre fini) sont topologiquement équivalentes si et seulement si elles ont les mêmes valeurs propres. Il arrivera très vite à généraliser l'invariant de Reidemeister au cas où l'action des groupes de rotations sur $S^{n}$ n'est pas libre et à démontrer que si $R_{2}=f R_{1} f^{-1}$ pour un difféomorphisme $f$, alors $R_{1}$ et $R_{2}$ ont bien les mêmes valeurs propres, [7]. Mais il n'arrivera jamais à démontrer ce résultat dans le cas où $f$ n'est qu'un homéomorphisme. Il y avait, pour cela, une bonne raison.

\section{Le contre-exemple de Cappell et Shaneson}

Dans la note [2] Cappell et Shaneson annoncent des résultats inattendus sur le problème d'homéomorphie des rotations, qui font l'objet de l'article [3]: Il existe deux rotations non isométriques sur la sphère $S^{9}$ qui sont homéomorphes. Considérons en effet les matrices de rotation suivantes, pour $\varepsilon= \pm 1$ :

$$
\frac{1}{\sqrt{2}}\left(\begin{array}{cccccccccc}
\varepsilon & \varepsilon & 0 & 0 & 0 & 0 & 0 & 0 & 0 & 0 \\
-\varepsilon & \varepsilon & 0 & 0 & 0 & 0 & 0 & 0 & 0 & 0 \\
0 & 0 & \varepsilon & \varepsilon & 0 & 0 & 0 & 0 & 0 & 0 \\
0 & 0 & -\varepsilon & \varepsilon & 0 & 0 & 0 & 0 & 0 & 0 \\
0 & 0 & 0 & 0 & \varepsilon & \varepsilon & 0 & 0 & 0 & 0 \\
0 & 0 & 0 & 0 & -\varepsilon & \varepsilon & 0 & 0 & 0 & 0 \\
0 & 0 & 0 & 0 & 0 & 0 & \varepsilon & \varepsilon & 0 & 0 \\
0 & 0 & 0 & 0 & 0 & 0 & -\varepsilon & \varepsilon & 0 & 0 \\
0 & 0 & 0 & 0 & 0 & 0 & 0 & 0 & -\sqrt{2} & 0 \\
0 & 0 & 0 & 0 & 0 & 0 & 0 & 0 & 0 & \sqrt{2}
\end{array}\right)
$$

Ces deux matrices de rotation (dans $S O(10)$ ) ont des traces différentes, et ne peuvent donc être linéairement équivalentes, mais elles décrivent des rotations de $S^{9}$ qui sont homéomorphes! De Rham écrira peu après la publication de ces travaux l'article historique [9] dont nous avons déjà parlé dans l'introduction.

\section{Références}

[1] Alexander, J.W.: Note on two threedimensional manifolds with the same group. Amer. Math. Soc. Transl. 20 (1919), 339-342.

[2] Cappell, S.E.; Shaneson, J.L.: Linear algebra and topology. Bull. Amer. Math. Soc. (N.S.) 1 (1979) 4, 685-687.

[3] Cappell, S.E.; Shaneson, J.L.: Nonlinear similarity. Ann. of Math. (2) 113 (1981) 2, 315-355

[4] de Rham, G.: Sur la dualité en analysis situs. C.R. Acad. Sci. Paris 186 (1928), 670-672.

[5] de Rham, G.: Sur l'analysis situs des variétés à $n$ dimensions. J. Math., IX. Ser. 10 (1931), 115-200.

[6] de Rham, G.: Sur les nouveaux invariants topologiques de M. Reidemeister. Rec. Math. [Mat. Sbornik] N.S. 1 (1936), 737-743.

[7] de Rham, G.: Reidemeister's torsion invariant and rotations of $S^{n}$. Differential Analysis, Bombay Colloq, Oxford Univ. Press, London 1964, 27-36.

[8] de Rham, G.: Quelques souvenirs des années 1925-1950. Cahiers du Séminaire d'Histoire des Mathématiques. 1 (French), Inst. Henri Poincaré, Paris, 1980, 19-36. 
[9] de Rham, G.: Homéomorphie des rotations de $S^{m}$. Évolution d'un problème, souvenirs. Enseign. Math. (2) 27 (1981) 3-4, 177-184.

[10] Franz, W.: Über die Torsion einer Überdeckung. J. Reine Angew. Math. 173 (1935), 245-254.

[11] Kuiper, N.H.; Robbin, J.W.: Topological classification of linear endomorphisms. Invent. Math. 19 (1973), 83-106.

[12] Reidemeister, K.: Homotopieringe und Linsenräume. Abh. Math. Sem. Univ. Hamburg 11 (1936), 102 109.

[13] Tietze, H.: Über die topologischen Invarianten mehrdimensionaler Mannigfaltigkeiten. Monatsh. Math. Phys. 19 (1908), 1-118.

Manuel Ojanguren et Jérôme Scherer

IGAT EPFL

CH-1015 Lausanne, Suisse

e-mail: manuel.ojanguren@epfl.ch

jerome.scherer@epfl.ch 